\title{
Modelling Microstructure and Properties during Annealing of Cold- rolled Al-Mn-Fe-Si-alloys with different Microchemistries
}

\author{
Knut Marthinsen ${ }^{1, a}$, Ning Wang ${ }^{1, b}$, and Ke Huang ${ }^{1, c}$ \\ ${ }^{12}$ Norwegian University of Science and Technology, N-7491 Trondheim, Norway

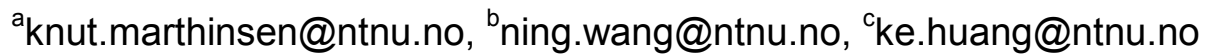

Keywords: Recovery, recrystallization, concurrent precipitation, Zener drag, modelling.

\begin{abstract}
The physical basis and a mathematical formulation of a softening model nicknamed Alsoft, accounting for the combined effect of recovery and recrystallization during annealing of heavily deformed aluminium alloys have been presented. The prediction power of the model is tested against experiments in terms of softening kinetics and final grain structure of selected Al-MnFe-Si-model alloys with different as homogenized microchemistries in terms solid solution levels of $\mathrm{Mn}$ (potential of concurrent precipitation) and different constituent particle and dispersoid structures. It is demonstrated that good model predictions may be obtained for alloys and conditions which are not or too a limited extent influenced by particle drag effects and concurrent precipitation while conditions strongly affected by these effects are increasingly difficult to model and in the most extreme cases impossible with reasonable input model parameters.
\end{abstract}

\section{Introduction}

To control mechanical properties of a material subjected to an annealing treatment after cold rolling, an adequate quantitative description of the softening behavior is crucial. The final annealing treatment is commonly carried out in order to obtain an optimum combination of strength and ductility, thus controlling the softening behavior is very important. This is particularly important for alloys prone to precipitation of dispersoids during processing, as pre-existing and/or concurrently precipitated dispersoids may strongly influence recovery and recrystallization kinetics and may have a significant effect on the final grain size and texture of the alloys [1,2].

Within the Norwegian aluminium community a softening model, nick-named Alsoft, which accounts for the combined effect of static recovery and recrystallization during annealing after hot/cold deformation, has been developed. The model has successfully been applied to predict the softening behaviour of various Al-alloys, in particular after hot deformation and conditions of mainly iso-thermal annealing [3-5]. In the present work the Alsoft model is applied and its prediction power discussed in relation to experimental data provided for the back-annealing behaviour of selected Al-Mn-Fe-Si-alloys presented recently [6-9]. It has clearly been shown that the softening behaviour during back annealing of cold rolled Al-Mn(-Fe-Si) alloys is the result of a critical balance between the processing conditions and microchemistry and its associated changes during processing. Following an increased use of recycled aluminium, typically, alloying elements like $\mathrm{Mn}, \mathrm{Fe}$ and Si will accumulate in secondary alloys, and generally give alloys with a broader variety in chemical composition and variations in microchemistry. It is there of great importance to possibly validate the Alsoft model for such alloys and compositions and to identify critical issues which needs to be the addressed in further developments.

\section{The Alsoft model}

The Alsoft model is based on a two-parameter description of the as-deformed sub-structure after cold/hot deformation where the microstructure is characterized by an average sub-grain size $\delta$ and a dislocation density $\rho_{i}$ inside the sub-grains. The average sub-grain size after deformation can be obtained from experiments or from adequate models [10]. During annealing of the as-deformed 
state recovery will take place through sub-grain growth and by annihilation of the sub-grain interior dislocations. Originally recovery kinetics was assumed to be controlled by solute drag only, where the rate controlling mechanism is assumed to thermal activation of solute atoms away from climbing jogs. In the present work, focusing annealing under conditions of possible strong dispersoid effects which may exert a strong Zener drag, the sub-grain growth expression is modified accordingly. Based on these assumptions evolution equations for sub-grain size and dislocation density can be expressed as follows [4,5]:

$$
\begin{aligned}
& \frac{d \rho_{i}(t)}{d t}=-v_{D} b A_{\rho} B_{\rho} \rho_{i}(t)^{3 / 2} \exp \left(-\frac{U_{a}}{R T(t)}\right) 2 \sinh \left(\frac{A_{\rho} G b^{4} \sqrt{\rho_{i}(t)}}{k T(t)}\right), \quad A_{\rho}=w_{\rho} c_{s s}^{-e} . \\
& \frac{d \delta(t)}{d t}=v_{D} b A_{\delta} B_{\delta} \exp \left(-\frac{U_{a}}{R T(t)}\right) 2 \sinh \left(\frac{A_{\delta}}{k T(t)}\left(\frac{G b^{4}}{\delta(t)}-P_{Z}(t) \frac{b^{3}}{\alpha_{4} \theta}\right)\right), \quad A_{\delta}=w_{\delta} c_{s s}^{-e} .
\end{aligned}
$$

Here $G$ is the shear modulus, $b$ is Burgers vector $(b=0.286 \mathrm{~nm}$ in aluminium $), \gamma_{S B}$ is the subboundary energy, $v_{D}$ is the Debye frequency, $k$ is Bolzmann's constant, $w_{\rho, \delta}$ and $e_{\rho, \delta}$ are model parameters, and $B_{\rho, \delta}$ alloy specific fitting constants. $c_{s s}$ is an effective level of solute atoms derived from summation of the solute concentration of the individual alloy elements, weighted with their activation energy for diffusion. $U_{a}$ is an activation energy, which in the case of solute drag equals that of diffusion of the relevant solute. $P_{Z}$ is the Zener drag due to dispersoids, which for spherical particles of mean size $r_{p}$ and volume fraction $f_{p}$ are defined by the last term in Eq. 3 below [1]. The effective instantaneous stored energy/driving pressure for recrystallization due to dislocations and sub-grains is calculated according to the following equation:

$$
P_{D}^{e f f}(t)=\alpha \frac{\gamma_{S B}}{\delta(t)}+\frac{1}{2} G b^{2} \rho_{i}(t)-\frac{3}{2} \frac{f_{p}(t) \gamma_{G B}}{r_{p}(t)}
$$

where $\gamma_{S B}$, the sub-boundary energy, may is obtained by the Read-Shockley equation [1].

The recrystallization module of Alsoft is an extension of the classical Johnson-MehlKolmogorov-Avrami $(J M A K)$ approach, treating recrystallization as a nucleation and growth process $[1,3]$. Nucleation of recrystallization assumed to take place from sub-grains which fulfil the general nucleation criteria for recrystallization, i.e. sub-grains which fulfil the Gibb's Thomson relationship; $\delta^{*}(t)>4 \gamma_{G B} / P_{D}^{\text {eff }}(t)$ and are surrounded by a high-angle boundaries, i.e. at deformation heterogeneities in the material where this is the case. In the current version of Alsoft, three such nucleation mechanisms are considered, i.e. from deformation zones around large particles (PSN), nucleation from old grain boundaries and nucleation from retained cube bands $[3,5]$.

The recrystallization kinetics is calculated by applying the standard assumptions of site saturation nucleation kinetics and a random distribution of nucleation sites, i.e. in which case the following transformation kinetics equation is obtained:

$$
\frac{d X(t)}{d t}=(1-X(t)) N_{t o t} 4 \pi \cdot r(t)^{2} \cdot G(t)
$$

Here $X(t)$ is the fraction recrystallized after an annealing time $t, r(t)$ is the size and $G(t)$ the growth rate of the a recrystallized nuclei/grain, i.e:

$$
\frac{d r}{d t}=G(t)=M(t) \cdot P_{D}^{e f f}(t) ; \quad M(t)=\frac{M_{0}}{c_{s s} k T} \exp \left(-\frac{U_{R X}}{R T}\right)
$$


$M_{0}$ is a modelling parameter and $U_{R X}$ is an activation energy which will depend on the alloy composition. An effect of solute drag is included through the inverse proportionality to $c_{s s}(a t \%)$. The grain size of a fully recrystallized structure is simply given by $D=\left(1 / N_{T o t}\right)^{1 / 3}$, with the total density of nucleation sites $N_{\text {Tot }}=N_{\text {Cube }}+N_{G B}+N_{P S N}$.

Eqs. 1-5 provides four differential equations to be solved in combination to give the time evolution of the relevant quantities, in principle for any temperature-time schedule. The result is used to calculate the associated yield stress during back-annealing given by the following relationship [4]:

$$
\sigma_{y}(t)=\sigma_{0}(t)+\left[\alpha_{1} M G b \sqrt{\rho_{i}(t)}+\alpha_{2} M G b \frac{1}{\delta(t)}\right][1-X(t)]
$$

where $\alpha_{1}$ and $\alpha_{2}$ are constants, with typically values of 0.3 and 2.5 , respectively. $\sigma_{0}$ is the yield stress of the fully soft condition expressed through $\sigma_{0}(t)=\sigma_{i}+\sigma_{s s}(t)+\sigma_{p}(t)$ where $\sigma_{s s}^{\text {eff }}(t)$ is the strength contribution from atoms in solid solution (incl. a base level accounting for impurities) and $\sigma_{p}(t)$ the contribution from non-shearable particles (primary particles and/or dispersoids).

\section{Application of the model}

The experiments serving as reference for model predictions refer to cold rolling and backannealing of two Al-Mn-Fe-Si-model alloys (C1 and $\mathrm{C} 2$ ) differing in the nominal amount of $\mathrm{Mn}$ (Table 1). As-received material (i.e. DC-cast extrusion billets) were subjected to two different homogenization treatments (A and $\mathrm{B}$ ) to provide materials with different microchemistries in terms of primary particle structures, dispersoid structures and solute level of Mn. Both procedures are designed to give considerable precipitation (i.e. limited $\mathrm{Mn}$ left in solid solution prior to final annealing) where condition A should give a course dispersoid structure, i.e. relatively low Zener drag, while the B-variant is designed to give a high density of small dispersoids, i.e. providing a much stronger Zener drag both during recovery and recrystallization (nucleation as well as growth).

Table 1. Two homogenization procedures and resulting concentration levels of $\mathrm{Mn}$ in solid solution

\begin{tabular}{|c|c|c|}
\hline Sample & Composition $(w t \%)$ & Homogenization \\
\hline $\mathrm{C} 1-\mathrm{A}$ & $0.5 \mathrm{Fe}+0.15 \mathrm{Si}+0.4 \mathrm{Mn}$ & $50^{\circ} \mathrm{C} / \mathrm{h}$ to $600^{\circ} \mathrm{C} / 4 \mathrm{~h} @ 600^{\circ} \mathrm{C} /(-) 25^{\circ} \mathrm{C} / \mathrm{h} / 4 \mathrm{~h} @ 500^{\circ} \mathrm{C}+$ quenching \\
\hline $\mathrm{C} 1-\mathrm{B}$ & $0.5 \mathrm{Fe}+0.15 \mathrm{Si}+0.4 \mathrm{Mn}$ & $50{ }^{\circ} \mathrm{C} / \mathrm{h}$ up to $4500^{\circ} \mathrm{C} / 4 \mathrm{~h} @ 450{ }^{\circ} \mathrm{C}+$ quenching \\
\hline $\mathrm{C} 2-\mathrm{B}$ & $0.5 \mathrm{Fe}+0.15 \mathrm{Si}+1.0 \mathrm{Mn}$ & $50^{\circ} \mathrm{C} / \mathrm{h}$ to $6000^{\circ} \mathrm{C} / 4 \mathrm{~h} @ 600^{\circ} \mathrm{C} /(-) 25^{\circ} \mathrm{C} / \mathrm{h} / 4 \mathrm{~h} @ 500^{\circ} \mathrm{C}+$ quenching \\
\hline $\mathrm{C} 2-\mathrm{B}$ & $0.5 \mathrm{Fe}+0.15 \mathrm{Si}+1.0 \mathrm{Mn}$ & $50^{\circ} \mathrm{C} / \mathrm{h}$ up to $450{ }^{\circ} \mathrm{C}+4 \mathrm{~h} @ 450{ }^{\circ} \mathrm{C}+$ quenching \\
\hline
\end{tabular}

The homogenized materials were cold rolled to a strain $\varepsilon=3.0$ before isothermal annealing at different temperatures and times. Back-scatter electron (BSE) images obtained in a scanning electron microscope (SEM) were used to characterize constituent particles and dispersoids in different stages of processing as well as the deformation sub-structure. The softening and precipitation behaviour during annealing were followed by Vickers hardness and electrical resistivity measurements as well as light optical microscopy (LOM) and SEM-EBSD to characterize the grain structure evolution. The experimental results are reported in detail elsewhere [7-10], together with more extended experimental work including also two homogenization variants which also gave conditions strongly affected by concurrent precipitation.

As far as possible experimentally measured relevant material parameters (e.g. alloy composition, as-cast grain size) and microstructure parameters (size and number density of primary particles and dispersoids) have been used as input. In line with the required input to the Alsoft model, the 3D cumulative size distribution of primary particles has been characterized according to the equation 
$F(\eta)=N_{0} \exp (-L \eta)$, where the parameters $N_{0}$ and $L$ have been determined by a least squares fitting to the experimental results. Relevant material and model parameters are given in Table 1, referring to the initial as-deformed state, from which $P_{D}$ and $P_{Z}$ are calculated from Eq.3, ignoring the contribution from sub-grain interior dislocations (amounting typically to only 1-2\%). Thermolectrical Power (TEP) measurements have been used to estimate the amount of Mn solid solution after the different homogenization treatments, and basis for the effective solute levels given in Table 1. In addition to the data given in Table 1, the time-temperature schedule during annealing can be explicitly specified on input, together with the corresponding time evolution of the effective solute level and the Zener drag following changes in the dispersoid structures [6-9]

Table 2 Actual material and model model parameters used in the Alsoft calculations

\begin{tabular}{|l|l|l|l|l|l|l|l|}
\hline & C1-3-3 & C1-2-3 & C1-2-3 & C2-3-3 & C2-2-3 & C2-2-3 & C2-2-3 \\
\hline \multicolumn{1}{|c|}{$T$} & All T & All T & $\mathrm{T}=300$ & All T & 350 & 400 & 450 \\
\hline Css_eff & 0.0012 & 0.0014 & 0.0014 & 0.0021 & 0.0023 & 0.0023 & 0.0023 \\
\hline $\mathrm{P}_{\mathrm{D}}(\mathrm{MPa})$ & 0.75 & 0.75 & 0.75 & 0.8 & 0.75 & 0.75 & 0.75 \\
\hline$\sigma_{0}(\mathrm{MPa})$ & 30 & 30 & 30 & 50 & 63 & 63 & 63 \\
\hline $\mathrm{N}_{0}\left(\# / \mathrm{m}^{3}\right)$ & $5.0 \mathrm{E}+16$ & $1.1 \mathrm{E}+17$ & $1.1 \mathrm{E}+17$ & $5.0 \mathrm{E}+16$ & $1.1 \mathrm{E}+17$ & $1.1 \mathrm{E}+17$ & $1.1 \mathrm{E}+17$ \\
\hline $\mathrm{L}\left(\mu \mathrm{m}^{-1}\right)$ & 2.02 & 2.78 & 2.78 & 2.02 & 2.78 & 2.78 & 2.78 \\
\hline $\mathrm{M}_{0}$ & $1.0 \mathrm{E}+05$ & $1.0 \mathrm{E}+05$ & $1.0 \mathrm{E}+05$ & $1.0 \mathrm{E}+05$ & $4.0 \mathrm{E}+04$ & $2.0 \mathrm{E}+04$ & $4.0 \mathrm{E}+04$ \\
\hline $\mathrm{P}_{\mathrm{Z}}(\mathrm{MPa})$ & 0.0 & 0.03 & $0.1^{*}$ & 0.075 & $0.12 / 0.5^{*}$ & $0.12 / 0.5^{*}$ & $0.12 / 0.5^{*}$ \\
\hline
\end{tabular}

* Parameter values used in the Alsoft simulations

Comparisons with experiments and modelling results are made in terms of the softening behaviour (yield stress versus time) and final recrystallized grain size, with the following conversion of hardness (VHN) to yield strength (YS): $Y S(M P a)=4.5 * V H N-85$ [11].

The model predictions for the softening behaviour of the $\mathrm{C} 1$ alloy, following the two homogenizations (A and B) together with the corresponding experimental results are shown in Figs. 1a and $\mathrm{b}$, respectively. Although the experimental results are scarce and somewhat scattered the calculated softening curves for C1-A seems to compare quite well with the experimental results.
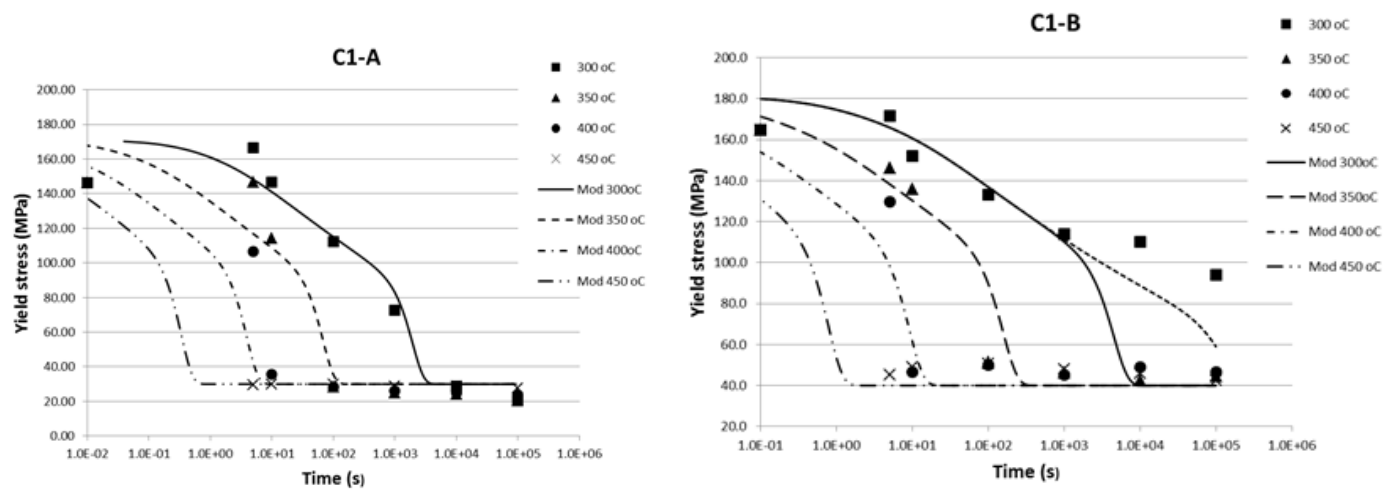

Fig. 9 Experimental softening curves and corresponding model predictions for the $\mathrm{C} 1$ alloy after different homogenization treatments during annealing after cold rolling to strain of $\varepsilon=3$.

Also for the second variant (C1-B, Fig. 1b), which is moderately affected by pre-existing dispersoids (cf. Table 2), the model predictions are mainly satisfactory. The exception is the lowest temperature where a considerable discrepancy is observed for larger times, where a fully soft condition is not reached, even with $10^{5} \mathrm{~s}$ of annealing. From the electrical conductivity measurements reported in [8], it is clear that the C1-B condition also experience some additional concurrent precipitation during annealing, an effect which is most pronounced at the lowest annealing temperature. In terms of modelling, a somewhat better agreement is obtained with a 
combination of a much lower mobility pre-factor $M_{0}$ and a tripled $P_{Z}$ value (dashed line). The predicted recrystallized grain sizes as presented Table 3, and compares favourably with the experiments, except for $\mathrm{C} 1-\mathrm{B}\left(300^{\circ} \mathrm{C}\right)$ which does not become fully recrystallized

The corresponding results for the C2-alloy with the higher amount of $\mathrm{Mn}(1 \mathrm{wt} \%)$ are shown in Fig. 2. Also in this case, the model predictions for the A variant, except perhaps for the lowest annealing temperature for long annealing times, are quite good, also with respect to grain size (Cf. Table 3). However, the C2-B condition posed more difficulties in reproducing the experimental results. With a much higher amount of $\mathrm{Mn}$, this condition contains a higher density of pre-existing dispersoids, and experience thus a significantly stronger Zener drag. The relevant material and model input parameters are given in Table 2. In particular it is noted that a considerably higher initial Zener drag (acting during nucleation) is needed (4x nominal) to give reasonable kinetics and grain size (Table 3), and consistent results were not obtained with the same parameters for all conditions.
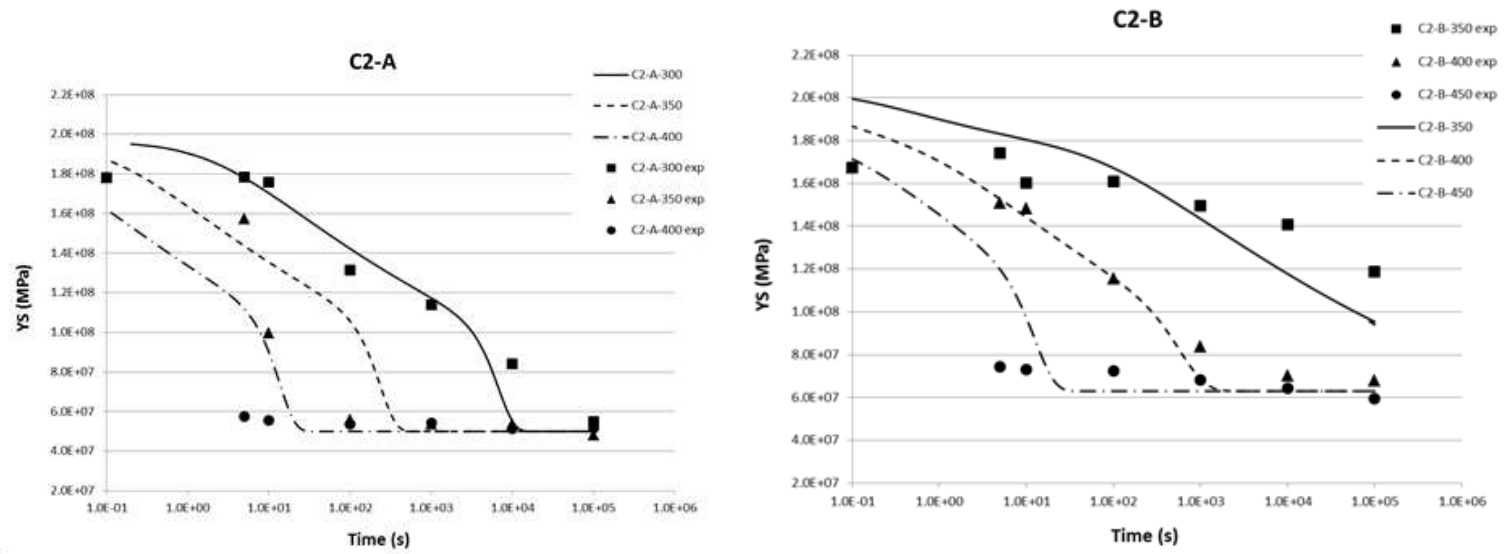

Fig. 2 Experimental softening curves and corresponding model predictions for the $\mathrm{C} 2$ alloy after different homogenization treatments during annealing after cold rolling to strain of $\varepsilon=3$.

Table 3 Calculated and experimentally measured grain sizes (circle area equiv. diameter)

\begin{tabular}{|l|l|l|l|}
\hline Alloy/condition & Anneal. temp. & Experiment $[\mu \mathrm{m}]$ & Model $[\mu \mathrm{m}]$ \\
\hline C1-A & All & 12 & 14 \\
\hline C1-B & All $\left(-300^{\circ} \mathrm{C}\right)$ & 22 & 26 \\
\hline C2-A & $450 / 400 / 350$ & $24 / 19 / 23$ & 19 \\
\hline C2-B & 450 & 83 & 88 \\
\hline
\end{tabular}

Even more difficulties in reproducing the experimental results are experience for homogenization condition No. 2 (i.e. C2-2; same as for C1-2 alloy), with a large amount of fairly small pre-existing dispersoids, i.e. a considerable Zener drag acting both during nucleation and growth. The relevant material and model input parameters are given in Tables 1-3 in Appendix. In particular it is noted that a considerably higher initial Zener drag (acting during nucleation) needed to be included to give reasonable kinetics and grain size, and consistent results were not obtained with the same parameters for all conditions. The annealing behaviour at the lower temperatures and for the lower strain was not possible to model with reasonable input parameters and reasonable results.

Attempts to model experimental conditions for which concurrent precipitation play a more dominant role (not included here) have proven even more difficult. Conditions where both the recovery stage and recrystallization reaction are strongly influenced by concurrent precipitation, have not been possible to model satisfactory, even with extreme and unphysical values of some of the model parameters. 


\section{Summary}

The physical basis and mathematical formulation of a softening model accounting for the combined effect of recovery and recrystallization during back-annealing of heavily deformed aluminium alloys has briefly been reviewed.

The prediction power of the model has been tested against selected available softening behaviour results of Al-Mn-Fe-Si-alloys and processing conditions of which may experience strong dispersoid effects during back-annealing, either from pre-existing dispersoids or concurrent precipitation. It has been demonstrated that the model provide quite good predictions, with consistent model parameters, of material and process conditions which experience no or limited influence of pre-existing dispersoids and/or concurrent precipitation. Providing reasonable model predictions becomes increasingly challenging with increasing influence of dispersoid effects and concurrent precipitation, and for the most strongly affected conditions reasonable model predictions is not possible even with extreme (unphysical) changes in the model parameters.

The present work has clearly indicated the need for certain changes to the model and further developments. Alsoft in its current state is based on the highly idealised assumption of site saturated nucleation kinetics, which does not seem consistent with the very long incubation time for onset of recrystallization and the sluggish recrystallization reaction often observed. Recent experiments [], also support the view that this assumptions needs to be relaxed. The results and their analysis also indicate that a classical temperature independent Zener drag is not satisfactory to account for the effects observed and that a more sophisticated particle-boundary interaction mechanism may be needed.

Acknowledgement: Financial support by the Research Council of Norway (Project No: 193179/I40) and the industrial partners, Hydro Aluminium and Sapa Technology is gratefully acknowledged. NTNU, through the "Strategic Area Materials" is also gratefully acknowledged for financial support to postdoc Ke Huang.

\section{References}

[1] F.J. Humphreys and M. Hatherly, Recrystallization and Related Annealing Phenomena, 2nd Ed. Elsevier Science Ltd., Oxford, United Kingdom, 2004.

[2] S. Tangen, K. Sjølstad T. Furu, and E. Nes. Metallurgical and Materials Transactions 41A (2010) 2970-2983.

[3] H. E. Vatne, T. Furu, R. Ørsund, and E. Nes, Acta materialia 44 (1996) 4463-4473.

[4] J.A. Sæter, B. Forbord, H.E. Vatne, and E. Nes, Proc. 6th International Conference on Aluminum Alloys, ICAA-6 eds. T. Sato et al., JILM, Japan, 1998, pp. 113-126.

[5] O. Engler, L. Löchte, J. Hirsch, Acta Materialia 55 (2007) 5449-5463.

[6] N. Wang, J.E. Flatøy, Y. Li, K. Marthinsen, Transactions of Nonferrous Metals Society of China, Vol 22 (8) (2012) 1878-1883.

[7] N. Wang, YJ Li and K. Marthinsen. Materials Science Forum Vol. 753 (2013) 231-234.

[8] K. Huang, Y. Li, K. Marthinsen, this conference.

[9] N. Wang, Microstructure, microchemistry and properties during annealing of cold-rolled AlMn-Fe-Si-alloys - Experiments and Modelling. PhD-thesis, NTNU, Norway, 2013.

[10] E. Nes and K. Marthinsen, Materials Science and Engineering, Vol. A322 (2002) 176-193.

[11]K. Sjølstad, Deformaton and softening of commercial AlMn-alloys - Experiments and Modelling. PhD-thesis, NTNU, Norway, 2013 